\title{
Power Loss Reduction in Power System based on PSO: Case Study
}

\author{
Sameer Singh \\ Research Scholar \\ Department of \\ Electrical Engineering \\ Jaipur National University, \\ Jaipur \\ Rajasthan, India
}

\author{
Vivek Kumar Jain \\ Asst. Prof./ Ph.D. Scholar \\ Department of \\ Electrical Engineering \\ Jaipur National University, \\ Jaipur \\ Rajasthan, India
}

\author{
Upendra Prasad \\ Professor \& Head \\ Department of \\ Electrical Engineering \\ B.I.T., Sindri, Dhanbad \\ Jharkhand, India
}

\begin{abstract}
In this paper, a new evolutionary approach has been discussed for reactive power dispatch (loss reduction) with the contribution of particle swarm optimization. Proposed algorithm has been applied to achieve the major objective as the system loss minimization with satisfied equality and inequality constraints. Tap settings of transformer, voltage at generator bus and shunt capacitor banks have been considered as control variables. Successful application of proposed algorithm is done on different IEEE bus systems. In comparison of other previous work, this proposed algorithm provides the better results.
\end{abstract}

\section{Keywords}

Reactive power optimization, particle swarm optimization, genetic algorithm and loss reduction

\section{INTRODUCTION}

Objective function is determined by the requirement of reactive power dispatch (loss reduction) at different location. Reactive power dispatch problem makes considerable effect on power system operation which results in loss in transmission system. It has considered as most prominent problem in the area of power system. The solution is made in reactive power dispatch in order of determining the location reactive power generation for optimization of objective function. Reactive power loss can also be responsible for the power factor decrement [1-3].

A stochastic, population based search technique termed as particle swarm optimization (PSO) is well suitably implemented for multi-dimensional space. Increased standard of living of people, industrialization, rural electrification at large scales lead to increase the demand of electric power in higher order in the world. The demand is dominating the supply [4]. To fulfill this criteria, establishment of renewed system for generation, transmission and distribution is required whereas decrement in losses caused technically such as in transmission, distribution system and the losses due to in-efficient metering, energy thefts should be made in order to fill the gap of supply and demand of energy as well as avoid the unwanted shutdowns. As the loss of the system would be maintained i.e. technical and non- technical both, utilization of available energy resources would be increased to satisfy the demand for the affordable transmission and generation cost. This requirement motivates to initiate the optimal power flow program for the planning and operation of a power system [5].Identification of appropriate control variable with allocation of their corresponding operating value is the main objective of an optimal power flow problem having a specific objective of loss minimization in the network with satisfied equality and inequality constraints. Derivation of equality constrains is done from the power balance equation whereas limits on control variables are the parameters to achieve in equality constraints that may be continuous or discrete in nature.

For solving problems like optimal power flow, economic load and reactive power dispatch/loss reduction etc., some traditional approaches such as newton method, quasi-newton, sequential linear programming, sequential quadratic programming and gradient method were employed [6]. On the basis of rapid convergence, easy handling of inequality constraints, problem solving ability for absence of initial feasible point, some available methods like deterministic methods, interior point method were taken into consideration over others. Interior point method involves the simplex method to find out improved direction striated in the feasible space with the movement from one to adjacent feasible vertex along the boundary of the feasible space till the achievement of the optimum point. As the various classical approaches were proposed, out of these some conventional optimization methods were considered for reliable solution of reactive power dispatch (loss reduction) problems, whereas in this case also global optimal solution has not guaranteed, while handling of constraints and discrete variable were the major issues for most of them. Initial selection condition decides the solution quality and global convergence [7]. For these methods requirement of presence of a differentiable and continuous objective function is noticed which availability is rare for practical reactive power dispatch (loss reduction) problems.

In past few decades, some artificial intelligence optimization technique have been proposed for solving optimization problem to avoid the difficulty and problems of capability of global search for many conventional techniques like genetic algorithm, particle swarm optimization, ant-bee colony, evolutionary algorithm, bacterial foraging have been widely used to solve reactive power dispatch problems.

In this paper, PSO algorithm is applied to achieve better performance in comparison of others. Since particle swarm optimization is inspired by the physical phenomenon like bird flocking, fish schooling etc. this algorithm gives the better result in terms of reduction in higher order in losses compare to other algorithm. Hence by the application of particle swarm optimization algorithm voltage profile is 
improved because of loss reduction. Testing of effectiveness of particle swarm optimization algorithm done on different IEEE bus system and the result are shown in tabular format for clear vision of advantages of this algorithm.

\section{PROBLEM FORMULATION}

Maintaining the voltage quality and reactive power dispatch (loss reduction) is the main objective. Finding of control variables is also a main job such as reactive power effective devices, voltage at generator buses and transformer tap setting parameters. As the equality and inequality constraints has been taken into consideration, inequality constraints are the variables of generator reactive power fact devices constraints and tap setting constraints whereas equality constraints are the power flow equation equality constraints.

\subsection{Power Losses Reduction}

The reactive power dispatch (loss reduction) problem aims at minimizing the real power loss in a power system while satisfying the unit and system constraints. This goal is achieved by proper adjustment of reactive power variables like generator voltage magnitudes $\left(\mathrm{V}_{\mathrm{gi}}\right)$, reactive power generation of capacitor banks $\left(\mathrm{Q}_{\mathrm{ci}}\right)$ and transformer tap settings $\left(\mathrm{T}_{\mathrm{k}}\right)$

This is mathematically stated as:

$$
\operatorname{Min} F_{1}=P_{\text {Loss }} \sum_{k=1}^{n l} g_{k}\left[V_{i}^{2}+V_{j}^{2}-2 V_{I} V_{J} \cos \left(\delta_{i}-\delta_{j}\right)\right]
$$

The real power loss given by (1) is a non-linear function of bus voltages and phase angles which are a function of control variables.

\subsection{System constraints}

The real power loss given by (1) is a non-linear function of bus voltages and phase angles which are a function of control variables. The minimization problem is subjected to the following equality and inequality constraints:

\subsubsection{Equality constraints}

- Real Power Constraints

$$
\begin{gathered}
P_{G i}-P_{D i} V_{i} \sum_{j=1}^{N_{B}} V_{j}\left(G_{i j} \operatorname{Cos}\left(\delta_{i}-\delta_{j}\right)+B_{i j} \operatorname{Sin}\left(\delta_{i}-\delta_{j}\right)\right) \\
=0
\end{gathered}
$$

$\mathrm{i}=1,2 \ldots \ldots \mathrm{N}_{\mathrm{B}}$

$$
\begin{gathered}
\text { Reactive Power Constraints } \\
\begin{array}{c}
Q_{G i}-Q_{D i} V_{i} \sum_{j=1}^{N_{B}} V_{j}\left(G_{i j} \sin \left(\delta_{i}-\delta_{j}\right)-B_{i j} \cos \left(\delta_{i}-\delta_{j}\right)\right) \\
=0
\end{array} \\
\mathrm{i}=1,2 \ldots \ldots N_{B}
\end{gathered}
$$

\subsubsection{Inequality constraints}

- Bus Voltage magnitude contraints

$V_{i}^{\min } \leq V_{i} \leq V_{i}^{\max } ; i \in N_{B}$

- Transformer tap position contraints:

$t_{k}^{\min } \leq t_{k} \leq t_{k}^{\max } ; i \in N_{T}$

- Generator bus reactive power constraint

$Q_{g i}^{\min } \leq Q_{g i} \leq Q_{g i}^{\max } ; i \in N_{g}$
$Q_{c i}^{\min } \leq Q_{c i} \leq Q_{c i}^{\max } ; i \in N_{c}$

- $\quad$ Ttransmission line flow constraints

$S_{l} \leq S_{l}^{\max } ; l \in N_{B}$

- Generation capacity constraint

$P_{G i}^{\min } \leq P_{G i} \leq P_{G i}^{\max } ; i \in N_{B}$

- $\quad$ Power balance constraint

$\sum_{i=1}^{N B} P_{G i}=P_{D}+P_{L}$

$X_{i}^{\text {lim }}=X_{i}^{\max }$ if $X_{i}>X_{i}^{\max }$

$X_{i}^{\text {lim }}=X_{i}^{\text {min }}$ if $X_{i}<X_{i}^{\text {min }}$

\section{PREVIOUS APPROACHES}

\subsection{Nonlinear programming}

In nonlinear programming (NP) maximizing or minimizing of a nonlinear function takes place in context of bound, nonlinear or linear context, in which equalities or inequalities can be considered as constraints [4].

$$
\min f(x)
$$$$
\mathrm{X}
$$

\subsection{Newton's Method}

Newton's method goes a step beyond the simple gradient method and tries to solve the RPD by observing that the aim is to always drive

$$
\nabla \psi_{\mathrm{x}}=0
$$

Since this is a vector function, we can formulate the problem as one of findingthe correction that exactly drives the gradient to zero (i.e. to a vector, all of whose elements are zero). Suppose we wish to drive the function $\mathrm{g}(\mathrm{x})$ to zero. The function $\mathrm{g}$ is a vector and theunknown, $\mathrm{x}$ are also vectors. The $\nabla \psi_{\mathrm{x}}$ is a Jacobean matrix which has now second order derivatives is called Hessian matrix. Generally, Newton's method will solve for the correction that is much closer to theminimum generation cost in one cost in one step than would the gradient method.

\subsection{Simplex linear programming}

This method is employed to linear programming problem for maximization of objective function. The simplex method only to linear programming problems in standard form where the objective function was to be maximized. Extend of this procedure for linear programming problems for minimization of objective function [8-9].

$w=c_{1} x_{1}+c_{2} x_{2}+c_{3} x_{3}+\ldots c_{n} x_{n}$.

\subsection{The Lambda -Iteration Method}

In this iteration, a variable lambda is introduced for solving constraints optimization problem termed as Lagrange multiplier. Solving of lambda on hand is considerable by system equation solution. Iterative method is use to solve the equation to full fill the demand of satisfaction of inequality constraints [9-12].

a. Assumption of a suitable value of $\lambda(0)$ and the value should be greater than the largest intercept of the incremental cost characteristic of the various generators.

b. Compute the individual generations

c. Check the equality

$$
p d=\sum_{n=1}^{n} p n
$$


is satisfied.

d. If not, make the second guess $\lambda$ repeat above steps

\section{EVOLUTIONARY ALGORITHMS}

This paper deals with basically two evolutionary algorithms for solving reactive power dispatch (loss reduction) problem i.e. Genetic Algorithm (GA) and particle swarm optimization (PSO) to obtain the best result among them.

\subsection{Genetic Algorithm}

Genetic algorithms (GAs) were invented by John Holland in the 1960s and were developed with his students and colleagues at the University of Michigan in the (70s. Holland's original goal was to investigate the mechanisms of adaptation in nature to develop methods in which these mechanisms could be imported into computer systems.GA is a method for deriving from one population of "chromosomes" (e.g., strings of ones and zeroes, or bits) a new population [10-15]. The selection operator chooses those chromosomes in the population that will be allowed to reproduce, and on average those chromosomes that have a higher fitness factor (defined bellow), produce more offspring than the less fit ones. Crossover swaps subparts of two chromosomes, roughly imitating biological recombination between two single chromosome ("haploid") organisms; mutation randomly changes the allele values of some locations (locus) in the chromosome; and inversion reverses the order of a contiguous section of chromosome.

\subsection{PARTICLE SWARM ALGORITHM}

PSO was proposed by Russell Eberhart and James Kennedy, an electrical engineer socio-psychologist respectively in 1995. as the name implies Particle is termed as individual solution represented in m-dimensional vector and group of particle moving in multi-dimensional space in random direction is stated as Swarm whereas finding the best solution from existing one is considered as optimization [3].

So overall by combining these all Particle Swarm Optimization, it can be termed as a technique to search by a group of particle where each and every particle individual particle has information about each and every individual particle [9]. On that basis updating of data takes place. Each particles tries to move in its local best whereas its final destination to achieve global best solution for that

- In the solution space particle moves with its coordinates in association of fitness (best solution) which has attained by the particle so far. This value is termed as personal best, pbest.

- Best value attained so far by a particle with the closeness of that particle is considered as another best value that PSO track and termed as gbest.

- Basic PSO concept consist of the acceleration of every particle to achieve the personal best and global best locations by a random generated acceleration for each time steps of iterations as represented in fig 1 .

- Following information is used to modify and update the location by the each particle:

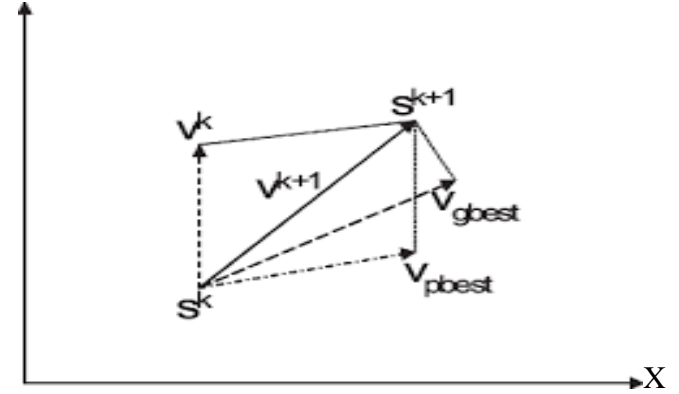

Fig 1: Modification Concept of a searching point by PSO

$$
\begin{gathered}
\mathrm{S}^{\mathrm{K}}: \text { Current searching point } \\
\mathrm{S}^{\mathrm{K}+1}: \text { Modified searching point } \\
\mathrm{V}^{\mathrm{K}}: \text { Current velocity } \\
\mathrm{V}^{\mathrm{K}+1} \quad: \text { Modified velocity } \\
\mathrm{V}_{\text {pbest }}: \text { Velocity based on } p_{\text {best }} \\
\mathrm{V}_{\text {gbest }}: \text { Velocity based on } g_{\text {best }}
\end{gathered}
$$

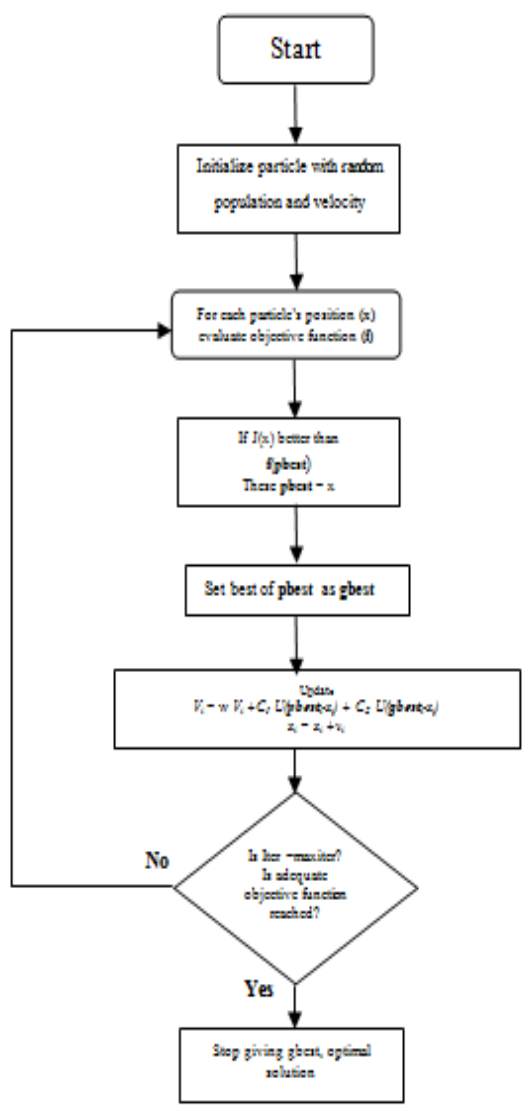

Fig 2: PSO algorithm

\subsubsection{PSO Algorithm}

- The current positions,

- The current velocities,

- The distance between the current position and pbest,

- The distance between the current position and the gbest.

Any optimization problem may be solved by generall particle swarm optimization algorithm. particle swarm optimization (PSO) algorithm has the basic pseudo-code is given as follows: 
Start

For each particle

Initialize particle with feasible random

numbers

End

While maximum number of iterations (or any other converge criterion) is not met

For each particle

Calculate fitness value

If the fitness value is better than best fitness value (pbest) in history

Set current value as the new pbest

End

End

Choose the particle with the best fitness value of all the particles in the history as the gbest

For each particle

Calculate particle velocity

Update particle position

\section{End}

\section{Finish}

\subsubsection{Velocity update mechanism}

$$
\mathrm{V}_{\mathrm{i}}^{\mathrm{k}+1}=\mathrm{wV}_{\mathrm{i}}^{\mathrm{k}}+\mathrm{C}_{1} \text { rand }_{1}(.) \times\left(\mathrm{p}_{\text {best }_{\mathrm{i}}}-\mathrm{s}_{\mathrm{i}}^{\mathrm{k}}\right)+\mathrm{C}_{2} \operatorname{rand}_{2}(.) \times\left(\mathrm{g}_{\text {best }}-\mathrm{s}_{\mathrm{i}}^{\mathrm{k}}\right)(17)
$$

$\mathrm{w}_{\mathrm{i}}=\mathrm{w}_{\text {max }}-\frac{\mathrm{w}_{\max }-\mathrm{w}_{\min }}{\text { iter }_{\max }} \times$ iter

Where,

$$
\begin{aligned}
& \mathrm{W}_{\max }=\text { initial weight } \\
& \mathrm{W}_{\min }=\text { final weight }
\end{aligned}
$$

Max. Iteration $=$ maximum iteration number

Iteration $=$ current iteration number

The current position (searching point in the solution space) can modified by the following equation

$s_{i}^{k+1}=s_{i}^{k}+V_{i}^{k+1}$

A local and global search is facilitated by the small and large inertia weight $(w)$ respectively. In comparison of fixed inertia weight setting, linear decrement of inertia weight from large to small value throughout the running of PSO provides the best performance result of PSO.

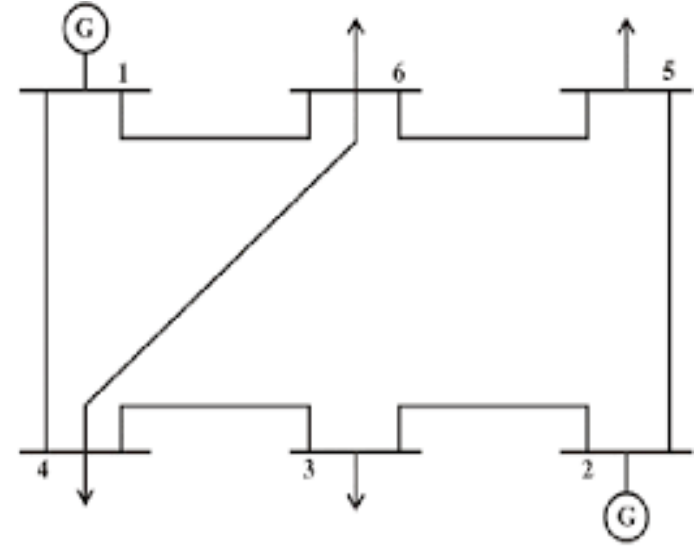

Fig 3: IEEE 6-Bus bus system

\section{RESULTS}

The IEEE bus system is used to show the practicability of the proposed algorithm and to novelty the optimal settings for generator voltages, transformer taps and switch-able reactive power sources. The algorithm is coded in MATLAB environment and a $2.30 \mathrm{GHz}$, Core i3, 4GB RAM based PC is for the simulation purpose.Parameters used in particle swarm optimization algorithm are (population size $=30$ constriction factor 0.71 ; number of iteration 100; acceleration constant $C_{1}=0.2$ and $C_{2}=0.2$; maximum and minimum velocity of particle $00.3 \& 00.3$; weight adaptive).

Table1: Types of buses with Variables

\begin{tabular}{|c|c|c|}
\hline Type of bus & $\begin{array}{c}\text { Known } \\
\text { Variable }\end{array}$ & $\begin{array}{c}\text { Unknown } \\
\text { Variable }\end{array}$ \\
\hline Load Bus & $\mathrm{PQ}$ & $\mathrm{V} \delta$ \\
\hline Slack Bus & $\mathrm{V} \delta$ & $\mathrm{PQ}$ \\
\hline Generator Bus & $\mathrm{PV}$ & $\mathrm{Q} \delta$ \\
\hline
\end{tabular}

Table 2: Control variables for IEEE 6-bus system

\begin{tabular}{|c|c|c|c|}
\hline \multirow{3}{*}{ Generator } & Variable & Min. & Max. \\
\cline { 2 - 4 } Voltage & $V_{1}$ & 1.00 & 1.10 \\
\hline \multirow{3}{*}{$\begin{array}{c}\text { Transformer } \\
\text { tap ratio }\end{array}$} & $V_{2}$ & 1.12 & 1.14 \\
\cline { 2 - 4 } & $T_{6}$ & 0.90 & 1.10 \\
\cline { 2 - 4 } & $T_{3}$ & 0.90 & 1.10 \\
\cline { 2 - 4 } & $T_{5}$ & 0.90 & 1.10 \\
\hline Var & $T_{4}$ & 0.90 & 1.10 \\
\cline { 2 - 4 } Support & $Q_{3}$ & 0.00 & 5.00 \\
\cline { 2 - 4 } & $Q_{6}$ & 0.00 & 5.00 \\
\hline
\end{tabular}

Table 3: Control variables results for IEEE 6-bus system before optimization

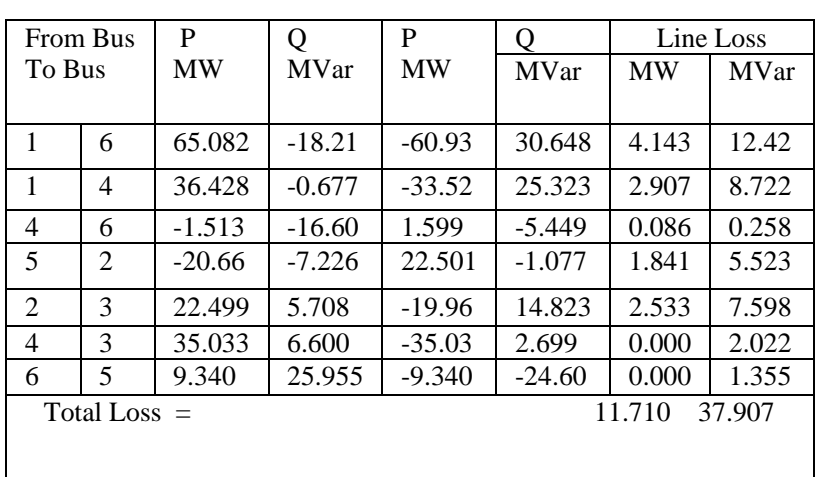


Table 4: comparison control variable settings before and after optimization using PSO for IEEE 6-bus system

\begin{tabular}{|c|c|c|l|}
\hline \multirow{4}{*}{$\begin{array}{c}\text { Generator } \\
\text { Voltage }\end{array}$} & Min. & Max. & $\begin{array}{c}\text { After } \\
\text { optimization }\end{array}$ \\
\cline { 2 - 4 } & 1.00 & 1.10 & 1.100 \\
\cline { 2 - 4 } & 1.12 & 1.14 & 1.094 \\
\hline \multirow{4}{*}{$\begin{array}{c}\text { Transformer } \\
\text { tap ratio }\end{array}$} & 0.90 & 1.10 & 0.973 \\
\cline { 2 - 4 } & 0.90 & 1.10 & 0.973 \\
\cline { 2 - 4 } & 0.90 & 1.10 & 0.973 \\
\cline { 2 - 4 } & 0.90 & 1.10 & 0.973 \\
\hline $\begin{array}{c}\text { Var } \\
\text { Support }\end{array}$ & 0.00 & 5.00 & 0.05 \\
\cline { 2 - 4 } & 0.00 & 5.00 & 0.05 \\
\hline
\end{tabular}

Table 5: Comparison of Power Loss in IEEE 6-bus System

\begin{tabular}{|c|c|l|l|l|}
\hline Loss (in & Loss (in & Loss (in & Loss (in & Loss \\
MW) & MW) & MW) & MW) With & (in \\
With & With & With & Genetic & MW) \\
NLP & NLP-IP & $\begin{array}{l}\text { Simplex } \\
\text { Method }\end{array}$ & Algorithm & With \\
& & $\begin{array}{l}\text { linear } \\
\text { method }\end{array}$ & & PSO \\
\hline 8.830 & 8.791 & 8.847 & 8.760 & 8.730 \\
\hline
\end{tabular}

Table 6: Power Loss results in IEEE6, 26 and IEEE 30 bus system

\begin{tabular}{|l|l|l|l|}
\hline Object & $\begin{array}{l}\text { IEEE6 BUS } \\
\text { SYSTEM }\end{array}$ & $\begin{array}{l}\text { IEEE26 } \\
\text { BUS } \\
\text { SYSTEM }\end{array}$ & $\begin{array}{l}\text { IEEE30 } \\
\text { BUS } \\
\text { SYSTEM }\end{array}$ \\
\hline $\begin{array}{l}\text { Loss (in } \\
\text { MW) }\end{array}$ & 8.730 & 19.865 & 5.808 \\
\hline
\end{tabular}

Figure 1 and figure 2 depict the concept of a searching point and PSO algorithm. Here the dependent variables depend on control variables. As losses are minimized, the power system will remain stable. Hence from simulation results shown, PSO optimization technique proves well with reduced losses.

\section{CONCLUSION}

In this paper, Particle Swarm optimization algorithm has been effectively applied to solve optimal reactive power dispatch (loss reduction) problem. The main objective is to minimize the active power loss in the network, while satisfying all the power system operation variable constraints (equality and inequality). The particle swarm algorithm has been coded using MATLAB software. The simulation results show that PSO algorithm always leads to a better result.

\section{ACKNOWLEDGMENTS}

The authors acknowledge the support and encouragement of Jaipur National University, Jaipur and BIT, Sindri, Dhanbad, Jharkhand, India.

\section{REFERENCES}

[1] Mamandur KRC and Chenoweth RD 1981. Optimal control of reactive power flow for improvements in voltage profiles and for real power loss minimization. IEEE Trans Power Appar Syst; PAS-100(7).

[2] Granville S. 1994. Optimal reactive dispatch through interior point methods, IEEE Trans. Power Syst., vol.9, no.1, pp.136-146.

[3] Kennedy J. 1997. The Particle Swarm: Social Adaptation of Knowledge. Proceedings of IEEE
International Conference on Evolutionary Computation, Indianapolis, USA, pp. 303-308.

[4] Saadat Hadi. 1999 "Power System Analysis", McGrawHill,.

[5] Chebbo M. and. Irving M. R. 1995. Combined active and reactive despatch \{Part 1: Problem formu- lation and solution algorithm, IEE Proc. Gener. Transm. Distrib. vol.142, no.4, pp.393-400.

[6] Shi Y, Eberhart R. 2000. Comparing inertia weights and constriction factors in particle swarm optimization. EvolComput; 1:84.

[7] Hirotaka Yoshida. 2000. "A Particle Swarm Optimization for Reactive Power and Voltage Control Considering Voltage Security Assessment", IEEE Transactions on Power Systems, Vol. 15, No. 4, (November 2000), pp. 1232-1239.

[8] Bhattacharyya B, Goswami SK. 2007. Combined heuristic and evolutionary approach for reactive power planning problem. J Electric Syst; 3 (4):203-13.

[9] Jain Vivek, Prasad Upendra. 2016. Solving Reactive Power Dispatch Problem using Evolutionary Computational Algorithm. ERCICA, Volume 03 ISBN 9789811002878, Chapter DOI $10.1007 / 9789811002878 \_44$ pp 470-481.

[10] Angeline P. J. 1998. Using selection to improve particle swarm optimization, Proceedings of IEEE Int. Conference on Evolutionary Computation,(May 1998). pp. 84-89

[11] Park Jong-Bae. 2006. Member, IEEE, Yun-Won Jeong, Woo-Nam Lee, and Joong-Rin Shin, "An Improved Particle Swarm Optimization for Economic Dispatch Problems with Non-Smooth Cost Functions," IEEE Power Engineering Society General Meeting,

[12] Jain Vivek k. and Prasad Upendra. 2015. Optimal reactive power dispatch based on particle swarm optimization. Journal of automation \& system engineering (JASE) - Vol. 9 | ISSN 1112-8542, pp258265.

[13] Devaraj D. and Preetha Roselyn J. 2010. Genetic algorithm based reactive power dispatch for voltage stabilityimprovement, Electrical Power and Energy Systems vol. 32, pp. 1151-1156.

[14] Thanushkodi K., Vijaya Pandian S. Muthu, Apragash R.S.Dhivy, Jothikumar M., sriramnivas S. and Vindoh K. 2008. An Efficient Particle Swarm Optimization for Economic Dispatch Problems With Non-smooth cost functions. WSEAS Transactions on Power Systems, Issue 4, Volume 3, (April 2008). , pp. 257-266

[15] Chen C. H. and Yeh S. N. 2006. Particle Swarm Optimization for Economic Power Dispatch with ValvePoint Effects. IEEE PES Transmission and Distribution Conference and Exposition Latin America, Venezuela, (Aug. 2006). pp. 15-18. 